Claremont Colleges

Scholarship@ Claremont

All HMC Faculty Publications and Research

HMC Faculty Scholarship

$1-1-2011$

\title{
Evidence of the Harmonic Faraday Instability in Ultrasonic Atomization Experiments with a Deep, Inviscid Fluid
}

Andrew P. Higginbotham '09

Harvey Mudd College

Aaron Guillen '11

Harvey Mudd College

Nathan C. Jones '10

Harvey Mudd College

Thomas D. Donnelly

Harvey Mudd College

Andrew J. Bernoff

Harvey Mudd College

\section{Recommended Citation}

A.P. Higginbotham, A. Guillen, N. Jones, T. D. Donnelly and A.J. Bernoff. Evidence of the harmonic Faraday instability in ultrasonic atomization experiments with a deep, inviscid fluid," J. Acoust. Soc. Am. 130 (2011) 2694-2699. doi: 10.1121/1.3643816

This Article is brought to you for free and open access by the HMC Faculty Scholarship at Scholarship @ Claremont. It has been accepted for inclusion in All HMC Faculty Publications and Research by an authorized administrator of Scholarship @ Claremont. For more information, please contact scholarship@cuc.claremont.edu. 


\title{
Evidence of the harmonic Faraday instability in ultrasonic atomization experiments with a deep, inviscid fluid
}

\author{
A. P. Higginbotham, A. Guillen, N. Jones, and T. D. Donnelly \\ Department of Physics, Harvey Mudd College, Claremont, California 91711 \\ A. J. Bernoff \\ Department of Mathematics, Harvey Mudd College, Claremont, California 91711
}

(Received 28 January 2011; revised 31 August 2011; accepted 1 September 2011)

\begin{abstract}
A popular method for generating micron-sized aerosols is to submerge ultrasonic $(\omega \sim \mathrm{MHz})$ piezoelectric oscillators in a water bath. The submerged oscillator atomizes the fluid, creating droplets with radii proportional to the wavelength of the standing wave at the fluid surface. Classical theory for the Faraday instability predicts a parametric instability driving a capillary wave at the subharmonic $(\omega / 2)$ frequency. For many applications it is desirable to reduce the size of the droplets; however, using higher frequency oscillators becomes impractical beyond a few MHz. Observations are presented that demonstrate that smaller droplets may also be created by increasing the driving amplitude of the oscillator, and that this effect becomes more pronounced for large driving frequencies. It is shown that these observations are consistent with a transition from droplets associated with subharmonic $(\omega / 2)$ capillary waves to harmonic $(\omega)$ capillary waves induced by larger driving frequencies and amplitudes, as predicted by a stability analysis of the capillary waves. (C) 2011 Acoustical Society of America. [DOI: 10.1121/1.3643816]
\end{abstract}

PACS number(s): 43.35.Pt [AJS]

Pages: 2694-2699

\section{INTRODUCTION}

Ultrasonic atomization is a versatile and popular technique for generating micron-scale aerosols. Aerosols produced in this manner have applications in industry, medicine, and nuclear fusion research. In many of these techniques the size of the ejected droplets is the key parameter. For example, the aerosols can be used to generate nanoparticles whose size depends on that of the ejected droplets, ${ }^{1}$ and appropriately sized droplets can be used as targets in laser-fusion experiments. ${ }^{2-5}$

The atomization technique involves submerging an ultrasonic piezoelectric transducer in a fluid bath. The transducer drives acoustic waves through the bath, which generates a capillary wave pattern at the fluid surface. If the ultrasonic waves are sufficiently intense, the capillary waves at the surface rupture, ejecting droplets and ultimately producing an aerosol.

Lang $^{6}$ and others ${ }^{4,5,7}$ established that the rupture of capillary waves at the fluid surface is responsible for the ejected droplets. Due to this observation, the problem of predicting the ejected droplet size is reduced to the determination of the dominant capillary mode at the surface of the excited fluid.

The observation of the surface patterns of a fluid acoustically excited at a frequency $\omega$ dates back to Faraday. ${ }^{8}$ While his experiments indicated a subharmonic $(\omega / 2)$ surface pattern, later experiments performed by Matthiessen ${ }^{9,10}$ suggested the possibility of a harmonic $(\omega)$ response. The discrepancy led Lord Rayleigh to consider the problem, ${ }^{11,12}$ but his observations indicated the presence of only subharmonic excitations. The issue did not rest on a firmer theoretical ground until a treatment by Benjamin and Ursell showed

\footnotetext{
a) Author to whom correspondence should be addressed. Electronic mail: donnelly@hmc.edu
}

that, for an inviscid fluid at low excitation amplitude, the subharmonic instability has a much larger growth rate than the harmonic instability. ${ }^{13}$

More recent work has focused on the possibility of exciting harmonic waves or solitary structures in a viscous fluid. ${ }^{14-18}$ However, the possibility of observing harmonic waves in the context originally considered by Benjamin and Ursell has received little attention.

Here we consider in detail the large drive amplitude structure of the theory developed by Benjamin and Ursell. We then present measurements of ejected droplet sizes which realize this structure. The measurements are well described by Benjamin and Ursell's original theory in the large drive amplitude regime.

\section{THEORY}

\section{A. Physics of atomization}

As originally proposed by Lang, ${ }^{6}$ the radius $r$ of a droplet ejected from an excited fluid surface should relate to the wavelength $\lambda$ of the ruptured wave. Following this intuition, the relation

$$
r=c \lambda / 2
$$

is introduced, where $c=0.34$ is an empirically determined constant. This relation has been experimentally verified over several orders of magnitude in dimensionless frequency. ${ }^{4-6}$ The problem of predicting the ejected droplet radius is thus reduced to determining the capillary wavelength $\lambda$ at the fluid surface.

This is accomplished with a linearized stability analysis of the fluid surface. ${ }^{4-6,13}$ The most unstable capillary wave initially grows the fastest, and is therefore assumed to 
dominate the aerosol production. The stability analysis relies on the results of Benjamin and Ursell, ${ }^{13}$ who considered the periodic, vertical excitation of a fluid container. This excitation is modeled as a parametric change in the gravitational constant $g$. In adopting this analysis for our experiment we are neglecting boundary and compressibility effects. This approximation is justified because both the container size $(100 \mathrm{~mm})$ and the acoustic wavelength $(\sim 1 \mathrm{~mm})$ are much larger than the capillary wavelength at the fluid surface $(\sim 1 \mu \mathrm{m})$.

According to this analysis, for large driving frequencies in an inviscid fluid, the initial small-amplitude oscillations $\eta$ at the fluid surface are governed by the Mathieu equation

$$
\frac{d^{2} \eta}{d T^{2}}+(p-2 q \cos (2 T)) \eta=0,
$$

where $q, p$ are nondimensional parameters and $T=\omega t / 2$. For a deep water bath, the parameter $p$ has the value $p=4 \omega_{k}^{2} / \omega^{2}$ where

$$
\omega_{k}^{2}=\sigma k^{3} / \rho
$$

is the frequency of a capillary surface wave, and $\sigma, \rho$ are the surface tension and density of the fluid, respectively. Note that in the limit of small forcing $p=1$ for subharmonic $(\omega / 2)$ excitation, while $p=4$ for the harmonic $(\omega)$ case. The parameter $q$ takes the value $q=2 k A$, where $k=2 \pi / \lambda$ is the surface wavenumber and $A$ is the driving amplitude. It is important to note that while this equation is valid only for small surface wave amplitude (i.e., soon after the vibrations begin $\eta \ll 1$ ), it remains valid even for large driving amplitudes $(A>1)$. Interestingly, increasing the drive amplitude does not increase the surface wave amplitude because droplet ejection occurs at wave amplitudes modestly beyond onset, thus limiting amplitude growth ${ }^{19,20}$ (see Sec. III C).

Using Lang's relation [Eq. (1)], one can obtain the observed droplet radius in terms of the most unstable $p$ value as

$$
r=\pi c\left(\frac{\sigma}{\rho p}\right)^{1 / 3}\left(\frac{2}{\omega}\right)^{2 / 3} .
$$

As shown by Benjamin and Ursell, for small drive amplitude (small $q$ ), one finds the most unstable mode is $p=1$. This result inserted in Eq. (4) is consistent with previous atomization work in the capillary regime. ${ }^{4-7}$ However, for larger drive amplitude this is not necessarily the case.

Figure 1 shows the growth rates for the subharmonic and harmonic modes as a function of the nondimensionalized drive amplitude $\alpha$. The growth rate $G$ is nondimensionalized such that the wave amplitude grows in proportion to $e^{G T}$. Mathematically, $G$ corresponds to the imaginary part of the Mathieu characteristic exponent. The nondimensionalized drive amplitude is defined such that ${ }^{21}$

$$
\alpha^{3}=2 \rho A^{3} \omega^{2} / \sigma
$$

For sufficiently large $\alpha$, the harmonic mode has a larger growth rate than the subharmonic. This corresponds to $p=4$ in Eq. (4), and thus would produce a decrease in droplet size by a factor of $4^{1 / 3} \approx 1.6$.

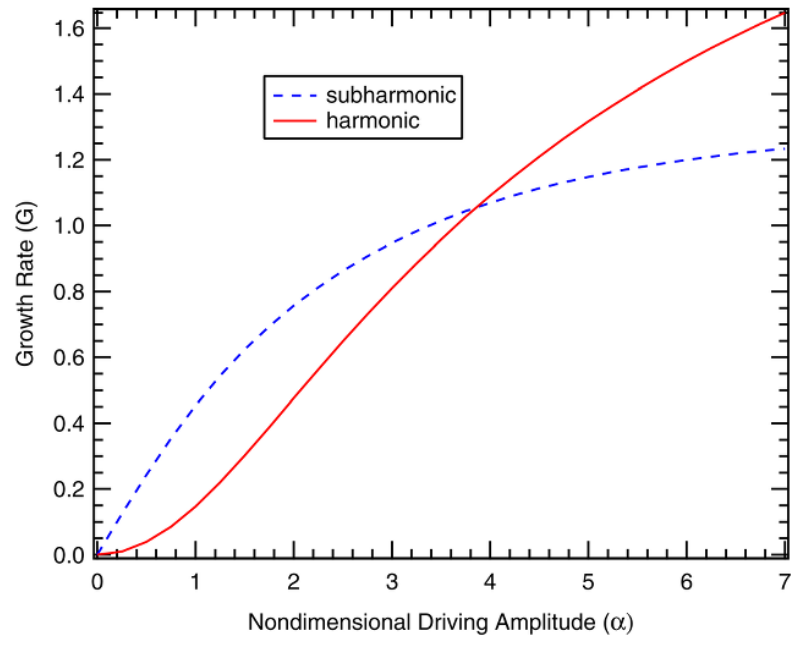

FIG. 1. (Color online) Onset growth rates for most unstable subharmonic and harmonic modes as a function of nondimensionalized driving amplitude $(\alpha)$. For large $\alpha$ the harmonic mode has a larger growth rate, and is therefore predicted to dominate the droplet ejection process.

For ultrasonic transducers in the $\mathrm{MHz}$ regime, wavelengths are on the order of $1 \mu \mathrm{m}$ and resonant transducer displacements on the order of $1 \mu \mathrm{m}$ are typical. ${ }^{22}$ Thus, for sufficiently large excitation frequencies, the possibility of atomization in the large $\alpha$ (harmonic) regime is worth considering.

\section{B. Stability diagram}

To understand the emergence of the harmonic Faraday instability suggested by Fig. 1 in further detail, it is useful to examine the stability diagram in Fig. 2. Each capillary wave is associated with a $(p, q)$ pair, and thus a point in the diagram. Waves with negative growth rate are shaded, and those with positive growth are white. The two white areas correspond to subharmonic and harmonic excitation, and the curves of maximum growth show the most unstable modes in each region.

Driving amplitude $(\alpha)$ enters into this picture through the dispersion relation Eq. (3), which can be rewritten

$$
p=(q / \alpha)^{3} \text {. }
$$

For small $\alpha$ one therefore obtains a dispersion curve which intersects the maximum growth curves at $p=1$ (subharmonic) and $p=4$ (harmonic). In this regime the subharmonic mode has the larger growth rate and is expected to dominate. For example, one can read off from Fig. 1 that when $\alpha=0.2$ the growth rate $G$ for subharmonic waves is $G=0.1$ while the growth rate for harmonic waves is $G=0.005$.

As $\alpha$ is increased the dispersion curve intersects the growth curves in a detuned region, which leads to weaker growth. For example, when $\alpha \approx 4$ the maximal subharmonic growth is substantially detuned from $p=1$ and the subharmonic mode begins to have a smaller growth rate than the harmonic (see Fig. 1).

This suggests harmonic droplet ejecting waves for sufficiently large $\alpha$. For the sake of concreteness, we label Fig. 2 with arrows to indicate a physically plausible path the 


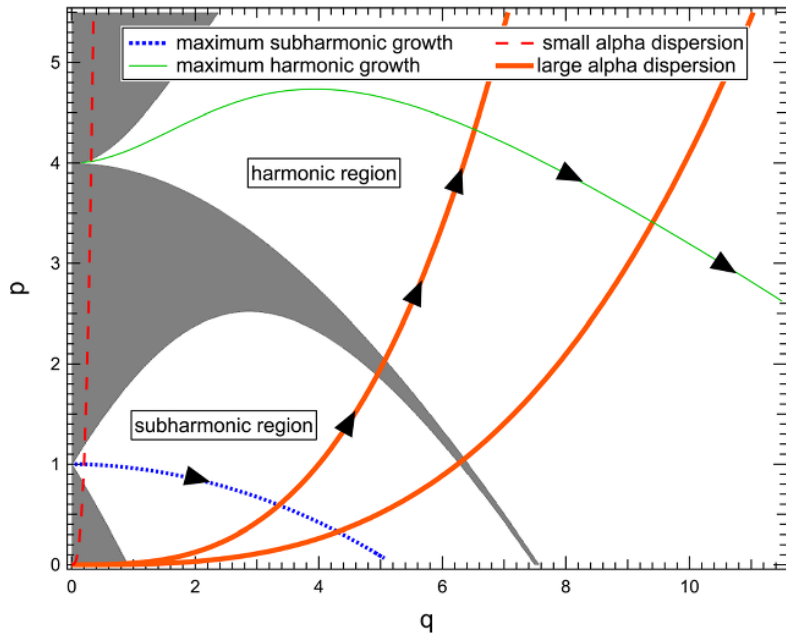

FIG. 2. (Color online) Stability chart for the Mathieu equation, Eq. (2). Points corresponding to negative growth rate are shaded, whereas the white tongues correspond to growth via subharmonic or harmonic excitation. For a fixed driving amplitude $\alpha$ the growth curves mark the most unstable modes in a particular regime. The curves increasing from the origin are the dispersion curves for the fixed alpha values $\alpha=0.2, \alpha=4$, and $\alpha=6.25$, and mark which modes may possibly be excited at the given drive amplitude $\alpha$. Thus the intersection of the growth and dispersion curves gives the most unstable mode in each regime. $\alpha=4$ is approximately where the harmonic growth rate is larger than the subharmonic, and $\alpha=6.25$ is where the original subharmonic $(\mathrm{p}=1)$ mode is no longer stable.

system could take to transition from the subharmonic to harmonic modes. However, in the context of the linearized theory it is not possible to comment on the nature of the transition from subharmonic to harmonic waves. Such a discussion would require an analysis of the competition between modes at the fluid surface, and thus the inclusion of nonlinear terms in the equations of motion.

To allow for the competition of several modes in the neighborhood of maximum growth we develop a conservative metric for classifying waves associated with the subharmonic and harmonic instabilities. We associate $p$ values from $p=1$ to $p=0.64$ with subharmonic waves. This range corresponds to $p$ values from the small a limit up to the point where the harmonic mode's growth rate exceeds that of the subharmonic, respectively. Similarly we associate $3.4 \leq p \leq 4.3$ with harmonic growth, where the lower and upper limits are the harmonic $p$ values for the two large $\alpha$ dispersion curves in Fig. 2. Using Eq. (4) with the appropriate $p$ value, harmonic and subharmonic Faraday excitations may be distinguished by measuring the droplet radius $r$ produced by an oscillator driven at a frequency $\omega$. For example, in an experiment with $2.5 \mathrm{MHz}$ excitation a droplet radius measurement of $r=1.1$ would be associated with subharmonic ejection whereas a measurement $r=0.7$ would be associated with harmonic ejection. We now describe our experimental efforts to realize such measurements in order to distinguish between subharmonic and harmonic ejection.

\section{EXPERIMENT}

\section{A. Method}

Examination of Eq. (5) reveals that $\alpha$ grows with both drive amplitude and frequency. From an experimental point of view, this suggests that the large $\alpha$ behavior we have described can occur for large excitation frequencies.

Aerosols were generated using high-frequency, piezoelectric oscillators submerged in the base of a $5 \mathrm{~cm}$ deep water bath. The oscillators were circular in shape, with a diameter of approximately $3 \mathrm{~cm}$, and the fluid was held in an acrylic cylinder with a $d=10 \mathrm{~cm}$ diameter. The large size of the cylinder $(d \gg \lambda)$ ensures that boundary effects may be ignored. The piezoelectric was driven with a sinusoidal voltage signal in the $\mathrm{MHz}$ range so that atomization occurred. Driving frequencies of 1.7, 2.5, 6.6, and 11.5 MHz were employed.

The ejected droplets were too small to be imaged directly $(\sim 1 \mu \mathrm{m})$, so a technique known as Mie scattering was used to accurately establish droplet size. In this approach the experimenter measures an angular scattering distribution using a monochromatic source and determines the size of the scatterer. The basic physics which makes Mie scattering a sensitive probe of droplet size in our experiment is that smaller droplets are closer to the Rayleigh limit and thus scatter more light into high angles.

A laser was directed into the aerosol, and the light scattered by the constituent droplets was measured using a photomultiplier tube mounted on a moveable arm. The arm was rotated through various angles $\theta$, generating an angular scattering pattern. The measured angular scattering pattern was normalized to the signal from a reference photomultiplier tube mounted at a fixed position. This procedure takes into account variations in laser intensity and aerosol density. The normalized angular scattering pattern corresponds uniquely to a droplet size distribution, which we determined using the theory of Mie scattering and a $\chi^{2}$ analysis.

Figure 3 shows the experimental setup. It is similar to the experiments we have previously used to characterize aerosols, ${ }^{4,5}$ with the exception that the new setup used a $1 \mathrm{kHz}, 60 \mathrm{fs}, 0.5 \mu \mathrm{J}$ Ti:sapphire laser as a light source. We found that the use of a pulsed laser system with a gated integrator greatly increased the sensitivity of our measurements.

\section{B. Analysis and discussion}

Sample angular scattering intensity profiles measured using the apparatus in Fig. 3 are presented in Fig. 4. The

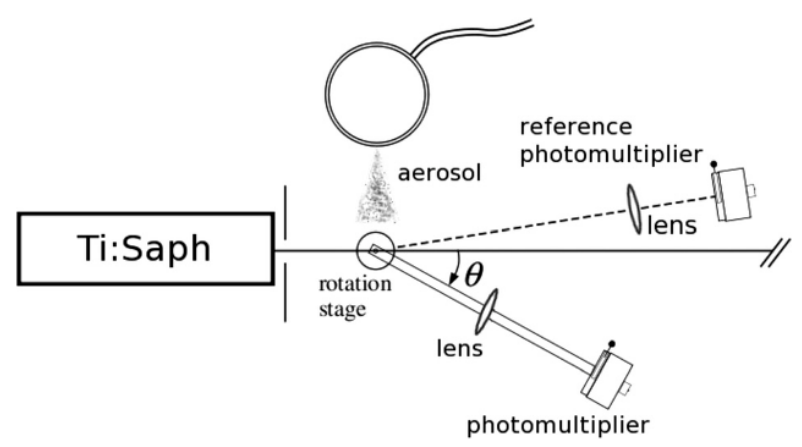

FIG. 3. Experimental setup. An aerosol was produced via ultrasonic atomization, and the constituent droplet size was then determined via Mie scattering. The aerosol scattered light from a Ti:sapphire laser, and an angular scattering pattern was measured using a photomultiplier tube mounted on a rotation arm. The signal is normalized to a reference detector to take into account variations in aerosol density and laser intensity. 


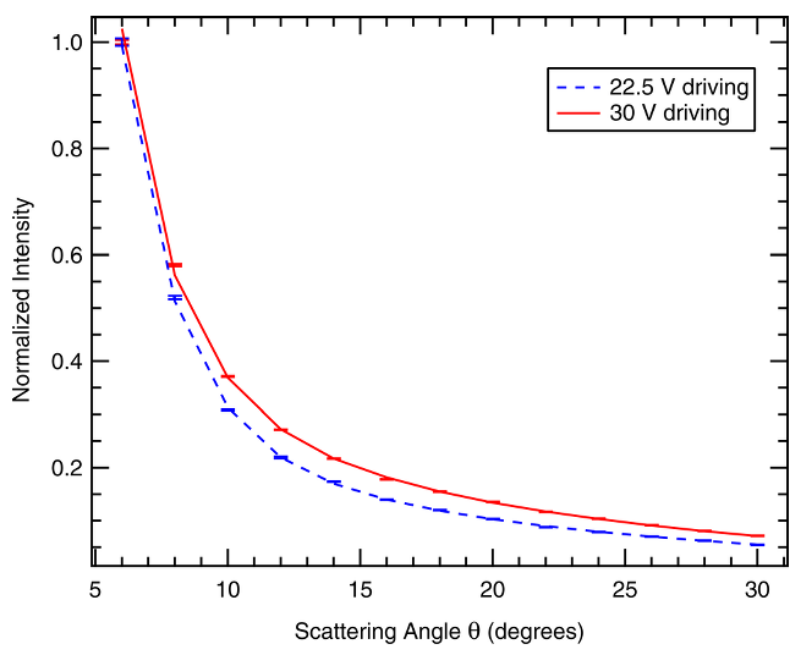

FIG. 4. (Color online) Typical Mie scattering curves from aerosols generated with a $2.5 \mathrm{MHz}$ oscillator at different drive amplitudes. Normalized intensity was measured using a photomultiplier tube as diagrammed in Fig. 3. The lines are calculated from a fit of the data using a $\chi^{2}$ analysis. Error bars are shown.

data shown in Fig. 4 were taken with a $2.5 \mathrm{MHz}$ oscillator at low $(22.5 \mathrm{~V})$, and high $(30 \mathrm{~V})$ driving amplitudes. The angular scattering patterns show a qualitative difference based on drive amplitude.

To analyze these angular scattering distributions, we model our experiment as the scattering of monochromatic plane waves due to a collection of Mie scatterers whose size follows a log-normal distribution. This size distribution allows for an asymmetric spread to take into account coalescence and evaporation, and is widely assumed in aerosol science. ${ }^{23}$ Allowing the mean and spread of the droplet distribution to vary, we determine the droplet size distribution which minimizes $\chi^{2}$ for the angular scattering data. As is shown in Fig. 4, this procedure yields good agreement with our observations.

The droplet distributions corresponding to the data in Fig. 4 are presented in Fig. 5. The mean value is $1.1 \mu \mathrm{m}$ $(0.6 \mu \mathrm{m})$ for $22.5 \mathrm{~V}(30 \mathrm{~V})$ driving.

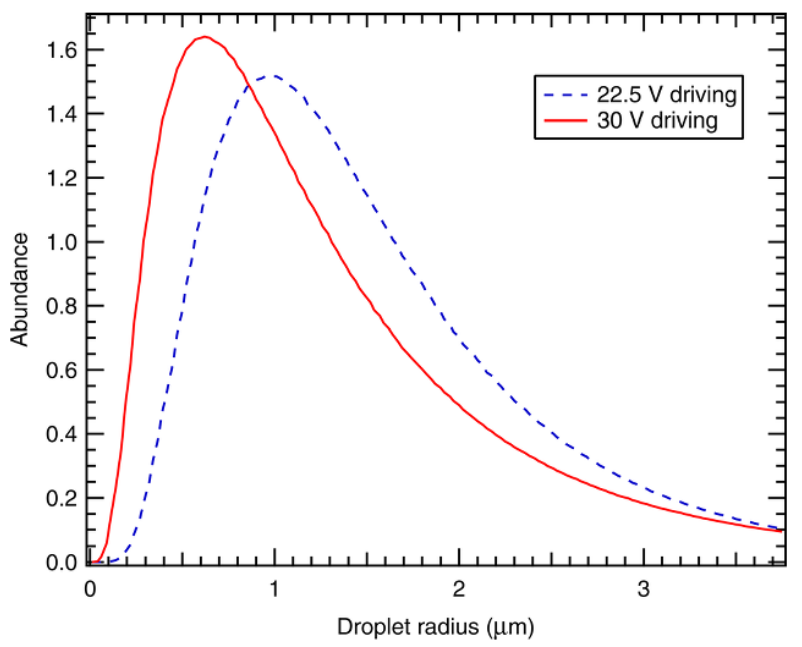

FIG. 5. (Color online) Droplet size distributions, calculated from a $\chi^{2}$ analysis of the data in Fig. 4.
The full data set is obtained by measuring ejected droplet radius for each oscillator at several drive amplitudes. Beginning with the $2.5 \mathrm{MHz}$ oscillator, we measured a range of driving voltages from 21 to $30 \mathrm{~V}$. At less than $21 \mathrm{~V}$ very little aerosol is generated, and above $30 \mathrm{~V}$ the piezoelectric may be damaged. The voltage was scanned by alternating between low and high drive voltages to eliminate the possibility of any long-term systematic errors. We then proceeded with the higher frequency measurements, returning at the end to a relatively low frequency $1.7 \mathrm{MHz}$ oscillator.

A compilation of all droplet sizes measured in this manner is presented in Fig. 6. The data are shown with the theoretical predictions of Eq. (4). The bands are derived from the ranges of $p$ values discussed in Sec. II B. The droplet sizes measured at $1.7 \mathrm{MHz}$ are associated with subharmonic waves, as has been found in other experiments. ${ }^{4,5}$ At a driving frequency of $2.5 \mathrm{MHz}$, as one would expect for a system at the boundary of the subharmonic and harmonic regimes, droplets consistent with both subharmonic and harmonic waves are observed depending on the drive amplitude. At 6.6 $\mathrm{MHz}$ and above, only droplets consistent with harmonic waves are observed. These observations agree with a transition to harmonic droplet ejecting modes at large $\alpha$.

The basic structure of these observations can be interpreted in the growth rate picture of Fig. 1 by recalling that $\alpha$ grows both with drive amplitude $A$ and excitation frequency $\omega$. The $1.7 \mathrm{MHz}$ oscillator has sufficiently small $\omega$ such that it is in the subharmonic regime for all feasible driving amplitudes $A$. The $2.5 \mathrm{MHz}$ oscillator has an intermediate $\omega$, which allows the system to be in either the subharmonic or harmonic dominated region of Fig. 1 depending on the drive amplitude $A$. The 6.6 and $11.5 \mathrm{MHz}$ oscillators have such large $\omega$ that the threshold drive amplitude for atomization occurs after the subharmonic and harmonic growth curves intersect.

Figure 7 more fully demonstrates the dependence of droplet size on driving voltage at the $2.5 \mathrm{MHz}$ excitation frequency. With the standard assumptions that our piezoelectrics

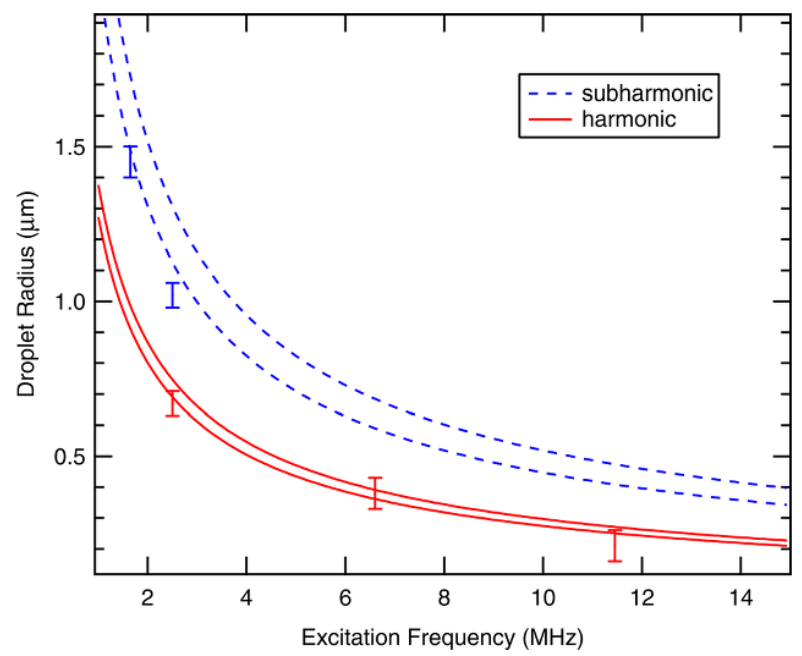

FIG. 6. (Color online) Droplet measurements with predictions from the modified linear theory. The bands are derived from the large $\alpha$ curves in Fig. 2. The error bars are derived from the standard deviation of the mean of multiple measurements. The theoretical curves have no free parameters. 


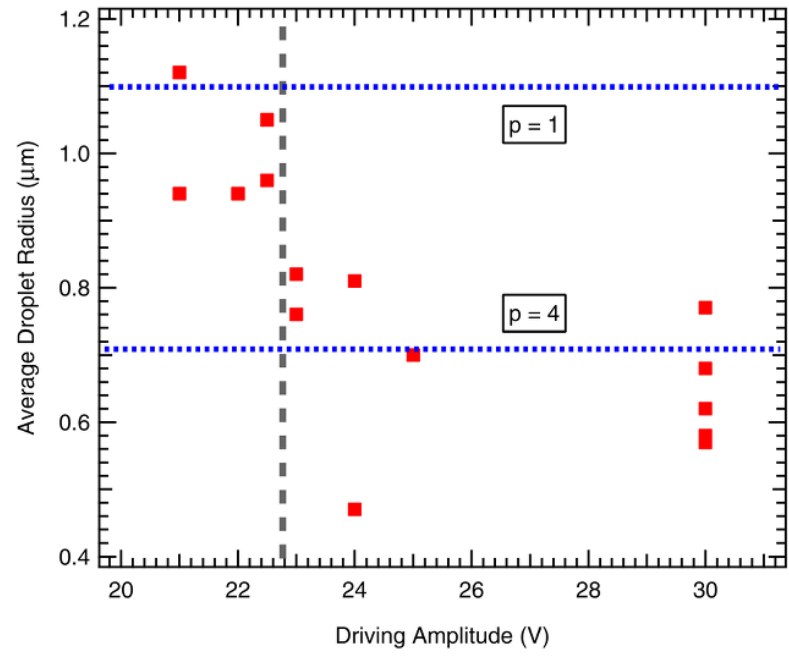

FIG. 7. (Color online) Measured droplet radius as a function of driving voltage for a $2.5 \mathrm{MHz}$ oscillator. For large drive amplitude, we observe smaller droplets. The dashed lines separate droplets we associate with the subharmonic and harmonic regimes. The horizontal bars correspond to the amplitude-independent predicted droplet sizes for $p=1$ and $p=4$.

obey a Hooke's law proportionality $V \propto A$ one can consider the driving voltage a proxy for the driving amplitude. The data therefore indicate a transition near a driving amplitude of $22.5 \mathrm{~V}$. We do not observe intermediate droplet sizes or a bimodal distribution during the transition. Also, the distributions show the same width before and after the transition. Thus, the data indicate a rather sharp shift in the mean droplet size. This is a striking qualitative feature, as the standard subharmonic theory of ultrasonic atomization predicts no dependence on drive amplitude.

It should be noted that the dispersion of the data in Figs. 6 and 7 is significantly larger than the uncertainties generated via $\chi^{2}$ analysis of the data in Fig. 4 . The origin of this dispersion could be physical, such as mode competition at the fluid surface. Therefore we use the mean of several droplet measurements and the standard deviation of the mean as the uncertainty.

\section{Validity of assumptions}

While we expect that nonlinear effects could weakly detune the resonant wavelength and the observed droplet size, droplet ejection occurs at wave amplitudes modestly beyond onset. ${ }^{19,20}$ Surprisingly, our use of large driving amplitudes and frequencies serves to reinforce this point. This is because droplets tend to be ejected when $h \sim \lambda,^{19}$ where $h$ is the height of the droplet ejecting wave. The wave height is therefore limited by the ejection process even as drive amplitude is increased. Additionally, the critical acceleration $a_{c}$ scales as $a_{c} \propto \omega^{4 / 3}{ }^{19}$ The droplet ejection height $h_{c}$ therefore scales as $h_{c} \sim \omega^{-2 / 3}$. As we use large $\omega$, this implies smaller surface amplitudes in our experiments than in previous ultrasonic atomization studies which are known to occur in a linear regime. ${ }^{4-6}$

Other mechanisms that could explain the driving amplitude dependence of droplet size, such as fluid heating and viscous effects, are unlikely. The temperature dependence of $\sigma$ and $\rho$ (Ref. 4) in Eq. (4), can change droplet radii by at most $0.05 \mu \mathrm{m}(\sim 0.5 \%)$. We have studied in detail the possibility of viscous effects in the droplet ejection process both numerically and experimentally, ${ }^{5}$ and have found that the parameters of this experiment are well within the inviscid regime. This is quantified through the computation of a nondimensional frequency $\Omega=\omega \eta^{3} / \rho \sigma^{2} \approx 10^{-3}$, where $\eta$ is the kinematic viscosity. Our numerical and experimental work shows that for $0<\Omega<0.2$ the inviscid approximation is valid.

\section{CONCLUSIONS}

We have presented our experimental finding of driveamplitude dependent droplet sizes in ultrasonic atomization experiments. These findings are explained in the context of the usual theory, which relates the ejected droplet size to the most unstable Faraday wave. The predicted transition in droplet sizes is observed-low frequency oscillators generate droplets associated with subharmonic Faraday waves while high-frequency oscillators generate droplet sizes consistent with a harmonic response. Intermediate frequency oscillators may excite either response based on their driving amplitude.

It would be interesting to gain more insight into the proposed transition from subharmonic to harmonic waves. Experimentally, one would like to probe this phenomenon in a lower frequency $(\mathrm{kHz})$ regime so that the surface waves might be directly imaged with a camera. In this regime piezoelectrics would likely no longer be appropriate due to their limited drive amplitudes. Theoretically, it would be interesting to examine in further detail the growth and mode selection mechanisms near the harmonic transition. Since for these experiments $a>g$ the Rayleigh-Taylor instability may play an important role. ${ }^{24}$ The inclusion of nonlinear terms in the equations of motion could also yield insights. From the point of view of applications, our observations open up new technical possibilities for the generation of small fluid droplets.

\section{ACKNOWLEDGMENTS}

This work was supported by the NSF Grant Nos. PHY0456898 and PHY-0757989 and partially by the American Chemical Society's Petroleum Research Fund.

${ }^{1}$ I. K. Wright, A. Higginbotham, S. M. Baker, and T. D. Donnelly, "Generation of nanoparticles of controlled size using ultrasonic piezoelectric oscillators in solution,” ACS Appl. Mater. Int. 2, 2360-2364 (2010).

${ }^{2}$ A. P. Higginbotham, O. Semonin, S. Bruce, C. Chan, M. Maindi, T. D. Donnelly, M. Maurer, W. Bang, I. Churina, J. Osterholz, I. Kim, A. C. Bernstein, and T. Ditmire, "Generation of Mie size microdroplet aerosols with applications in laser-driven fusion experiments," Rev. Sci. Instrum. 80, 063503 (2009).

${ }^{3}$ T. Donnelly, M. Rust, I. Weiner, M. Allen, R. Smith, C. Steinke, S. Wilks, J. Zweiback, T. Cowan, and T. Ditmire, "Hard X-ray and hot electron production from intense laser irradiation of wavelength-scale particles," J. Phys. B. 34, 313-320 (2001).

${ }^{4}$ T. Donnelly, J. Hogan, A. Mugler, M. Schubmehl, N. Schommer, A. Bernoff, S. Dasnurkar, and T. Ditmire, "Using ultrasonic atomization to produce an aerosol of micron-scale particles," Rev. Sci. Instrum. 76, 113301 (2005).

${ }^{5}$ T. Donnelly, J. Hogan, A. Mugler, N. Schommer, N. Schubmehl, A. Bernoff, and B. Forrest, "An experimental study of micron-scale droplet 
aerosols produced via ultrasonic atomization," Phys. Fluids 16, 2843-2851 (2004).

${ }^{6}$ R. J. Lang, "Ultrasonic atomization of liquids," J. Acoust. Soc. Am. 34, 6-8 (1962).

B. A. Puthenveettil and E. J. Hopfinger, "Evolution and breaking of parametrically forced capillary waves in a circular cylinder," J. Fluid Mech. 633, 355-379 (2009).

${ }^{8} \mathrm{M}$. Faraday, "On the forms and states assumed by fluids in contact with vibrating elastic surfaces," Philos. Trans. R. Soc. London 52, 319 (1831).

${ }^{9}$ L. Matthiessen, "Akustische versuche, die kleinsten transversalwellen der flüssigkeiten betreffend (acoustic experiments concerning the smallest transverse waves of fluids)," Ann. Phys. (Leipzig) 134, 107-117 (1868).

${ }^{10} \mathrm{~L}$. Matthiessen, "Ueber die transversalschwingungen tönender tropfbarer und elastíscher flüssigkeiten (on the transverse vibrations of an acoustically excited inviscid fluid)," Ann. Phys. (Leipzig) 141, 375 (1870).

${ }^{11}$ L. Rayleigh, "On maintained vibrations," Philos. Mag. 15, 229-235 (1883).

${ }^{12}$ L. Rayleigh, "On the crispations of a fluid resting upon a vibrating support,” Philos. Mag. 16, 50-58 (1883).

${ }^{13}$ T. Benjamin and F. Ursell, "The stability of the plane free surface of a liquid in vertical periodic motion," Proc. R. Soc. London 225, 505-515 (1954).

${ }^{14}$ H. W. Müller, H. Wittmer, C. Wagner, J. Albers, and K. Knorr, "Analytic stability theory for Faraday waves and the observation of the harmonic surface response,” Phys. Rev. Lett. 78, 2357-2360 (1997).
${ }^{15} \mathrm{~K}$. Kumar and L. S. Tuckerman, "Parametric instability of the interface between two fluids,” J. Fluid Mech. 279, 49-68 (1994).

${ }^{16} \mathrm{~K}$. Kumar, "Linear theory of Faraday instability in viscous liquids," Proc. R. Soc. London, Ser. A 452, 1113-1126 (1996)

${ }^{17}$ E. Cerda and E. Tirapegui, "Faraday's instability for viscous fluids," Phys. Rev. Lett. 78, 859-862 (1997).

${ }^{18} \mathrm{O}$. Lioubashevski, H. Arbell, and J. Fineberg, "Dissipative solitary states in driven surface waves," Phys. Rev. Lett. 76, 3959-3962 (1996).

${ }^{19}$ C. L. Goodridge, W. T. Shi, H. G. E. Hentschel, and D. P. Lathrop, "Viscous effects in droplet-ejecting capillary waves," Phys. Rev. E 56, 472-475 (1997).

${ }^{20}$ C. L. Goodridge, H. G. E. Hentschel, and D. P. Lathrop, "Breaking Faraday waves: Critical slowing of droplet ejection rates," Phys. Rev. Lett. 82, 3062-3065 (1999).

${ }^{21}$ The physical reason for the dependence on drive frequency is that the length scale of the problem is set by the capillary wavelength, and thus its frequency.

${ }^{22}$ P. Shashank, D. Viehland, A. Caraza, J. Ryu, and K. Uchino, "High-power resonant measurements of piezoelectric materials: Importance of elastic nonlinearies,” J. Appl. Phys. 90, 1469-1479 (2001).

${ }^{23} \mathrm{M}$. Kerker, The Scattering of Light and Other Electromagnetic Radiation (Academic, Waltham, MA, 1969), pp. 351-396.

${ }^{24}$ S. Kumar, "Mechanism for the Faraday instability in viscous liquids," Phys. Rev. E 62, 1416-1419 (2000). 